\title{
KOOPERTIONEN
}

\section{Betriebskindergärten als Win-win-Situation}

\author{
Sven Spier
}

\begin{abstract}
Die Zusammenarbeit zwischen freigemeinnützigen Trägern der Sozialen Arbeit und Wirtschaftsunternehmen beschränkt sich vielfach immer noch auf Sponsoring-und Corporate-Citizenship-Modelle. Dass auch Kooperationen im »Kerngeschäft « sozialer Organisationen möglich sind, zeigen Betriebskindergärten, die ein Gewinn für beide Partner sein können.
\end{abstract}

Die Betreuung, Erziehung und Bildung der jüngsten Kinder steht in Deutschland im Fokus der öffentlichen Aufmerksamkeit. Die Politik auf Bundes- und Landesebene hat erkannt, dass sie sowohl die Vereinbarkeit von Familie und Beruf als auch die Bildungschancen von Kindern durch einen verstärkten Ausbau der Kindertagesbetreuung nachhaltig fördern kann.

Vor diesem Hintergrund ist der massive Ausbau der Betreuung für Kinder im Krippen- und Kindergartenalter bis zum Jahr 2013 durch die liche Träger von Kindergärten, neue Betreuungsplätze schaffen - und sich hierfür mögliche Kooperationspartner zu suchen.

Diese Option offenbart den Charme, einen Betriebskindergarten $\mathrm{zu}$ gründen: Ein Unternehmen schafft für seine Mitarbeiterinnen und Mitarbeiter einen enormen Beitrag zur Work-life-Balance, steigert somit die Familienfreundlichkeit und kann dies nicht zuletzt als Standortfaktor bei der Gewinnung von gut qualifizierten Arbeitnehmern nutzen. Gleichzeitig erhält der Kindergartenträger die Möglichkeit, seine Fachlichkeit in ein wirtschaftliches Umfeld einzubringen. Zudem leistet ein Betriebskindergarten durchaus einen nicht geringen Beitrag zur ökonomischen Entwicklung des Unternehmens und des freien Trägers.

Unter dem Aspekt des Fachkräftemangels betrachtet zeigt sich, wie schwierig es derzeit in Deutschland noch ist, Kinder und Beruf zu vereinbaren. Während kinderlose Frauen in Deutschland

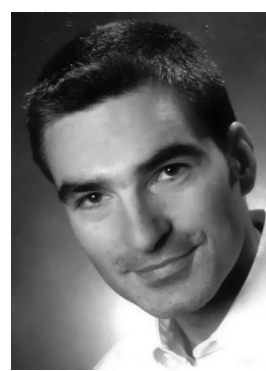

Sven Spier (36) hat in Braunschweig Sozialwesen und Sozialmanagement und in Hildesheim Erziehungswissenschaft, Politik und Betriebswirtschaftslehre studiert. Nach leitenden Tätigkeiten in der Jugendhilfe und einer mehrjährigen Tätigkeit als Referent für Kinder- und Jugendhilfe für einen Spitzenverband der Wohlfahrtspflege ist er nun Organisationsberater bei der Management- und Unternehmensberatung contec GmbH. E-Mailspier@contec.de

Hinzu kommt, dass derzeit neben der massiven Förderung durch den Bund auch von Landesseite der Aufbau neuer Betreuungsplätze erheblich unterstützt wird. In allen Bundesländern existieren hierzu Förderprogramme, die zum Teil eine großzügige finanzielle Unterstützung gewähren. Der Zeitpunkt, in den Bereich der (frühkindlichen) Betreuung einzusteigen, war selten von besseren Rahmenbedingungen begleitet und wir dies nach Ende der Ausbauperiode 2013 auch kaum wieder sein.

Durch Betriebskindergärten werden die Risiken für beide Seiten minimiert. Der freie Träger kann auf Ressourcen des Unternehmens zurückgreifen und die Belegung wird weitgehend gesichert. Das Unternehmen kann die Größe des Betriebskindergartens den vorhandenen und erwarteten Bedarfen anpassen. Darüber hinaus sind Kindergärten oder Horte in Betrieben gemäß einer aktuellen Untersuchung wirtschaftlich sinnvoll. So rechnen sich Betriebskindergärten in der Regel für Unternehmen mit mehr als 500 Beschäftigten, in denen hoch qualifizierte Mitarbeiter gebraucht werden oder dessen Arbeitsfeld, bzw. regionalem Einzugsgebiet Fachkräftemangel herrscht. Ein Betriebskindergarten erweist sich als wirtschaftlicher Vorteil, da die Neubesetzung eines hoch qualifizierten Arbeitsplatzes bis zu 25.000 Euro kosten kann. 


\section{Belegplätze: Wie ein Kindergartenträger und ein Wirtschaftsunternehmen kooperieren können}

Vertrag über die Anmietung von Belegrechten zwischen der Kinderbetreuung e. V.

und

dem Betrieb:

Anschrift:

Ansprechpartner/in:

Der oben genannte Betrieb beteiligt sich an »Kinderbetreuung e. V.« und eröffnet seinen Mitarbeiterinnen und Mitarbeitern die Möglichkeit, Kindergartenplätze zu nutzen. Er mietet bei dem Verein ab dem

Belegplätze für seine Mitarbeiterinnen und Mitarbeiter an. Die Anzahl der Plätze orientiert sich an dem Bedarf, der in der Belegschaft gegeben ist.

Es wird jedoch vereinbart, dass die Obergrenze der Plätze, die angemietet werden, bei Ganztagsplätzen Zweidrittelplätzen , Halbtagsplätzen und Teil-

zeitplätzen bei liegt.

\section{Kosten für den Betrieb}

Zusätzlich zum üblichen Elternanteil ist von der Firma eine Unterstützung zu leisten. Mit diesem Unterstützungssatz werden die Angebote finanziert, die bestehende Kindergärten nicht abdecken und die sich an dem besonderen Bedarf der Betriebe orientieren (zum Beispiel erweiterte Öffnungszeiten). Dieser Satz beträgt monatlich bei Vollzeitplätzen

Euro, Zweidrittelplätzen Euro, Halbtagsplätzen Euro und bei Teilzeitplätzen Euro.

\section{Kündigung des Belegplatzes}

Ein Belegplatz kann mit einer Frist von drei Monaten zum Ablauf des Kindergartenjahres gekündigt werden. Das Kindergartenjahr beginnt mit dem Tag, der auf das Ende der Sommerferien in folgt und endet am letzten Tag der Sommerferien des darauffolgenden Jahres. Die Kündigung hat schriftlich zu erfolgen.

\section{Anmietung von zusätzlichen Belegplätzen}

Sollte der Betrieb in Zukunft ein Interesse an der Anmietung weiterer Belegplätze haben, wird er gegenüber interessierten Unternehmen, die noch keine Belegplätze angemietet haben, bevorzugt behandelt. Die Mitarbeiterinnen und Mitarbeiter des Betriebes können sich jederzeit in die Warteliste des Kindergartens eintragen.

\section{Vereinbarungen zum Schutz der Kinder}

Grundsätzlich gilt die Anmeldung und Aufnahme eines Kindes bis zum Eintreten der Schulpflicht. Wird auch eine Schulkinderbetreuung im Anschluss gewünscht, so wird das Nutzungsrecht verlängert. Der Betrieb hat die Möglichkeit, mit Eintritt der Schulpflicht neu darüber zu entscheiden, ob er den Unterstützungssatz weiterhin leisten möchte. Sollte dies nicht der Fall sein, kann die Schulkinderbetreuung erfolgen, indem die Eltern den Unterstützungssatz in Eigenleistung erbringen. Das Recht auf Nutzung des Kindergartens wird nicht berührt durch die Auflösung des Arbeitsverhältnisses des Elternteils mit dem Betrieb. Der Betrieb hat, soweit er die Belegplatzmiete oder Teile davon getragen hat, die Möglichkeit, nach Auflösung des Arbeitsverhältnisses die Unterstützungszahlung in voller Höhe vom Mitarbeiter leisten zu lassen.

Ort, den

Für Kinderbetreuung e.V.

Für den Betrieb

Quelle: Bundesministerium für Familie, Senioren, Frauen und Jugend (Hg.): Unternehmen Kinderbetreuung. Praxisleitfaden für betriebliche Kinderbetreuung, 2008. 